\title{
Modelo de gestión de listas de espera centrado en oportunidad y justicia
}

\author{
CRISTIAN JULIO ${ }^{1,2, a}$, PATRICIO WOLFF ${ }^{1,2, b}$, MARÍA VEGOÑA YARZA ${ }^{2,3, \mathrm{c}}$
}

\section{Waiting lists management model based on timeliness and justice}

Waiting lists for elective surgery are considered a major health policy concern in most countries of the world. The most common reason to explain this phenomenon is that demand exceeds supply. Traditionally, the management of waiting lists has been focused on timeliness of medical attention. The objective of this paper is to present a waiting lists management model that includes the concepts of timeliness and justice simultaneously. We designed a prioritization method based solely on medical criteria. We developed a computer software to register patients, to prioritize and monitor the waiting lists. The system was implemented in 2013 and is currently used in all surgical specialties at a public hospital. The results show that timeliness does not suffice to manage the waiting lists for elective surgery, and therefore it should be complemented with an indicator of justice. Under this management model, hospitals should attempt to balance justice with timeliness of care and prioritize initiatives that improve both indicators at the same time. In addition, we propose using this model to manage the waiting lists of other hospital processes.

(Rev Med Chile 2016; 144: 787-794)

Key words: Economics, Medical; Health Policy; Hospitals, Public; Waiting Lists.

\section{Antecedentes}

\section{Listas de espera}

L a existencia de listas de espera se ha planteado como un problema mayor de políticas públicas de salud tanto en Chile como en países desarrollados ${ }^{1,2}$. En el contexto quirúrgico electivo, esta situación se agudiza si se considera que, en los últimos años, se ha observado un aumento de la demanda en países con sistema de salud público con alta cobertura en la población ${ }^{3}$.

La razón más recurrente para explicar la generación de listas de espera quirúrgicas es una demanda que excede a la oferta ${ }^{1-6}$.

Por el lado de la oferta, la capacidad de los sistemas públicos de salud está limitada en financiamiento, especialistas y otros recursos ${ }^{3,7-9}$. Una forma de enfrentar esta situación es priorizando la atención de problemas de salud que mejoren el resultado sanitario del sistema.

Por el lado de la demanda, existe evidencia que los costos asociados a la espera por una intervención quirúrgica están relacionados con efectos sociales, efectos sicológicos, grado de discapacidad, y con la probabilidad de deterioro de su condición de salud ${ }^{4,8,10-11}$. Este último riesgo puede disminuirse si se establecen criterios de oportunidad de atención para los pacientes. Bajo esta mirada, la existencia de listas de espera no es negativa per $s e$, sino que supone un desafío de gestión clínica para los hospitales públicos.

El objetivo de este trabajo es proponer un modelo de gestión de listas de espera que avance en la conciliación entre oferta y demanda en Chile, a través de una caracterización clínica de la lista $y$ un proceso de toma de decisiones que asegure oportunidad y justicia en la atención. 


\section{Urgencia, prioridad y justicia}

Los conceptos de urgencia, prioridad, y justicia, son ampliamente utilizados en listas de espera por cirugía electiva ${ }^{2-3,10,12}$.

El nivel de urgencia refiere al plazo dentro del cual debe atenderse al paciente antes que su estado de salud se deteriore significativamente; por lo tanto, corresponde a una definición paciente-céntrica. La oportunidad es la conveniencia del tratamiento de un paciente en un período temporal apropiado, por lo que este concepto se relaciona directamente con urgencia.

La prioridad representa la posición relativa de una persona en la lista de espera en un momento dado; es una mirada del paciente en contexto, donde su lugar en la lista puede construirse en función de la urgencia de atender su patología y la del resto de los pacientes esperando. Luego, el nivel de urgencia es centrado en un paciente mientras que la prioridad es centrada en todos los pacientes.

Según Evans \& Price, es posible definir tres tipos de justicia: igualitaria, que busca distribuir los recursos a las personas que más lo necesitan; utilitaria, que no se enfoca en la distribución sino más bien en la suma del resultado de las decisiones; y basada en méritos, que entrega recursos a las personas que más lo merecen bajo algún criterio predefinido $^{7}$. En el contexto de salud pública, el concepto igualitario de justicia implica favorecer a las personas en mayor necesidad de atención, mientras que el concepto utilitario se centra en maximizar los años de vida totales de la población por paciente tratado o por peso invertido.

Luego, el concepto de justicia está asociado con dos ámbitos de la lista de espera por cirugía electiva. Por una parte, con los criterios incluidos en la construcción del método de priorización, que pueden ser de carácter igualitario, utilitario, $o$ una combinación de ambos. Por otro lado, con la selección de pacientes, en tanto el criterio respeta en mayor o menor medida, su prioridad en lista de espera.

\section{Priorización de listas de espera en el mundo}

En la última década, países como Italia, Nueva Zelandia, Canadá, Gales, y Australia, han implementado sistemas de priorización de pacientes quirúrgicos electivos ${ }^{9-10,13-15}$. Generalmente, el diseño de estos sistemas responde al deseo de justicia, oportunidad, transparencia, y consistencia en las decisiones de ingreso y salida de la lista de espera $^{3,7,16-17}$.

Aún existe poca evidencia de aceptación de criterios para la priorización de pacientes y sobre la base ética más apropiada para hacerlo ${ }^{2,8-9}$. Aunque el consenso general es la inclusión de criterios clínicos como eje central de la priorización ${ }^{1}$, también se ha propuesto considerar factores físicos, psicológicos, y sociales del paciente $e^{3,4,8,13,18}$.

Uno de los métodos de priorización más utilizados es la clasificación de pacientes en categorías de urgencia, cada una de las cuales tiene asociado un tiempo máximo de espera para el tratamiento ${ }^{18}$. Estos métodos pueden tener distintos grados de formalización ${ }^{1}$ : sistemas menos formalizados proponen directrices para la clasificación de pacientes en cada categoría, mientras que otros más formales utilizan un algoritmo explícito.

Diversos autores han realizado una revisión de criterios de priorización de listas de espera para cirugía electiva $^{3-4,12}$. En general, existe consenso sobre la importancia de realizar tanto un proceso de validación clínica como de evaluación práctica del método de priorización que se decida utilizar ${ }^{12}$.

\section{Métodos para la priorización de pacientes}

\section{Categorización de pacientes}

El método propuesto en este trabajo consiste en clasificar los pacientes en categorías de urgencia con un tiempo máximo de espera asociado. La construcción de este método se realizó mediante la estructuración de conocimiento médico e incluye exclusivamente criterios clínicos; en este sentido, es consistente con una mirada igualitaria de justicia. Se definieron seis categorías de urgencia, cuyo detalle se muestra en la Tabla 1.

Tabla 1. Categorías de urgencia y tiempo máximo de espera

\begin{tabular}{|cc|}
\hline Categoría & Tiempo máximo de espera [días] \\
\hline AA & 0 \\
A & 15 \\
B & 30 \\
\hline C & 60 \\
\hline D & 180 \\
\hline E & 360 \\
\hline
\end{tabular}


El método de categorización considera dos elementos: el diagnóstico del paciente y sus agravantes. Cada diagnóstico puede tener asociado uno o más agravantes, que corresponden a condiciones y características del paciente que aumentan la gravedad de su patología. Por lo tanto, la construcción de este método requirió la definición de los diagnósticos más frecuentes de cada especialidad, la categoría de urgencia y agravantes asociados a cada diagnóstico, y cómo todas las combinaciones posibles de agravantes pueden cambiar -y en qué medida- la urgencia de atención del paciente con esa patología.

En las Tablas 2 y 3 se encuentran ejemplos de agravantes para distintos diagnósticos y aplicación del método a casos específicos, respectivamente.

\section{Priorización de lista de espera}

El método de priorización permite definir la posición de cada paciente en una lista de pacientes categorizados, con distintas complejidades biomédicas y tiempos máximos de espera. Para este trabajo se construyó una lista de espera por especialidad.

Tabla 2. Ejemplos de definición de agravantes asociadas a diagnósticos

\begin{tabular}{|c|c|}
\hline Diagnóstico & Agravantes \\
\hline Fimosis & $\begin{array}{l}\text { Daño renal } \\
\text { Antecedentes de parafimosis } \\
\text { Infección urinaria/Sepsis foco urinario } \\
\text { Obstrucción }\end{array}$ \\
\hline Hernia inguinal & $\begin{array}{l}\text { Edad menor a } 2 \text { años } \\
\text { Bilateralidad } \\
\text { Antecedentes de atascamiento }\end{array}$ \\
\hline
\end{tabular}

Tabla 3. Aplicación del método de categorización a casos específicos

\begin{tabular}{|lc|}
\hline Caso clínico & Categoría \\
\hline Fimosis - sin agravantes & E \\
\hline Fimosis con obstrucción & A \\
\hline Fimosis con antecedentes de parafimosis & D \\
\hline Hernia inguinal-sin agravantes & B \\
$\begin{array}{l}\text { Hernia inguinal en niño menor de } 2 \text { años y } \\
\text { con antecedentes de atascamiento }\end{array}$ & A \\
\hline
\end{tabular}

Primero, el método calcula un puntaje de prioridad para cada paciente, en base a: el tiempo máximo de espera asociado a su categoría; los días de espera; y un factor de vencimiento. La fórmula para calcular este puntaje se muestra a continuación:

Puntaje $_{P}=\left\{\begin{array}{l}\operatorname{Venc}_{C_{P}} \cdot\left(\text { De }_{P}-\operatorname{Dmax}_{C_{P}}\right), \operatorname{De}_{P}>\operatorname{Dmax}_{C_{P}} o C_{P}=A A \\ \left(\operatorname{De}_{P}-\operatorname{Dmax}_{C_{P}}\right)\end{array}\right.$

donde $C_{P}$ es la categoría del paciente $\mathrm{P} ; D e_{P}$ son los días de espera del paciente P; $\max _{C_{P}}$ son los días máximos de espera asociados a la categoría del paciente $\mathrm{P} ; \mathrm{Venc}_{C_{P}}$ corresponde al factor de vencimiento asociado a la categoría del paciente $\mathrm{P}$, que se utiliza cuando un paciente alcanza su tiempo máximo de espera.

El tiempo máximo de espera se determina en base al método de categorización presentado anteriormente. Los días de espera del paciente se calculan como los días trascurridos entre la indicación quirúrgica y el momento en que se consulta la lista de espera. El factor de vencimiento es un parámetro cuyo valor aumenta para categorías más urgentes. Estos dos últimos elementos otorgan un carácter dinámico a la lista de espera, ya que los pacientes con patologías más graves aumentarán de prioridad más rápido que aquéllos con problemas de salud de menor urgencia.

Finalmente, el método ordena los pacientes de mayor a menor puntaje. A nivel agregado, el resultado final es una lista de espera priorizada en base a criterios médicos estandarizados.

\section{Resultados}

Para implementar el proceso de priorización y selección de pacientes para cirugía electiva, se diseñó y desarrolló un sistema computacional que permite el ingreso de pacientes a la lista de cada especialidad y el seguimiento de todo el proceso de resolución quirúrgica. Es el sistema el que categoriza a los pacientes en base a parámetros definidos por los equipos médicos de cada especialidad.

Aunque los métodos de categorización y priorización propuestos han sido utilizados tanto en niños como en adultos ${ }^{19}$, en este trabajo se muestra su aplicación en el hospital pediátrico Dr. Exequiel González Cortés. Los resultados que se presentan 
Tabla 4. Lista de espera y distribución porcentual de pacientes

\begin{tabular}{|lcc|}
\hline Especialidad & Lista de espera mensual promedio & Distribución porcentual \\
\hline Cirugía general & 960 & $45 \%$ \\
\hline Cirugía plástica y quemados & 361 & $17 \%$ \\
\hline Urología & 339 & $16 \%$ \\
\hline Traumatología & 249 & $12 \%$ \\
Otras especialidades & 218 & $10 \%$ \\
\hline Total & 2.127 & $100 \%$ \\
\hline
\end{tabular}

Tabla 5. Distribución de categorías de urgencia por especialidad

\begin{tabular}{|c|c|c|c|c|c|c|}
\hline \multirow[t]{2}{*}{ Especialidad } & \multicolumn{6}{|c|}{ Distribución por categoría } \\
\hline & AA & $\mathbf{A}$ & B & C & D & $\mathbf{E}$ \\
\hline Cirugía general & $4 \%$ & $22 \%$ & $17 \%$ & $13 \%$ & $6 \%$ & $38 \%$ \\
\hline Cirugía plástica y quemados & $28 \%$ & $9 \%$ & $5 \%$ & $13 \%$ & $20 \%$ & $25 \%$ \\
\hline Urología & $18 \%$ & $6 \%$ & $12 \%$ & $20 \%$ & $11 \%$ & $33 \%$ \\
\hline Traumatología & $12 \%$ & $17 \%$ & $19 \%$ & $16 \%$ & $19 \%$ & $17 \%$ \\
\hline Otras especialidades & $2 \%$ & $7 \%$ & $5 \%$ & $36 \%$ & $45 \%$ & $5 \%$ \\
\hline
\end{tabular}

en esta sección fueron generados con información registrada entre junio de 2013 y julio de 2015.

En este análisis se incluyeron las cuatro especialidades con mayor demanda por cirugía electiva, que concentran cerca de $90 \%$ de las intervenciones del hospital. La Tabla 4 contiene el detalle de estas especialidades. Complementariamente, es posible caracterizar la incidencia de pacientes quirúrgicos a través de la distribución de sus categorías de urgencia. Esta información se muestra en la Tabla 5.

Por el lado de la oferta, el hospital tiene una capacidad de cuatro pabellones para cirugía electiva y un pabellón para el servicio de Urgencias. La Figura 1 muestra la asignación mensual promedio de pabellones por especialidad.

\section{Definición de oportunidad y justicia}

Para efectos de este trabajo, se utilizará la siguiente definición de oportunidad:

Oportunidad $_{t}[\%]=\frac{\text { Pacientes operados a tiempo }_{t}}{\text { Total de pacientes operados }}+100 \%$

Los pacientes operados a tiempo corresponde al número de personas que fueron operadas oportunamente en un intervalo $t$, y el total de pacientes operados $_{t}$ incluye todos los pacientes intervenidos en el mismo período. De esta forma, mientras mayor sea el número de pacientes operados a tiempo, entonces el indicador tendrá un valor más cercano a $100 \%$.

Por otra parte, se define justicia de la siguiente forma:

Justicia $_{t}[\%]=\left[1-\frac{\text { Prioridad promedio de pacientes operados }}{t}\right] \cdot 100 \%$

La prioridad corresponde al lugar en que se encuentra un paciente en la lista de espera. Por tanto, mientras más alta sea la prioridad promedio de los pacientes operados durante un período $t$, entonces el indicador tendrá un valor más cercano a $100 \%$ y se dirá que el proceso de selección de pacientes es más justo. Esta definición de justicia es consistente con una mirada igualitaria de la decisión de atención de pacientes.

\section{Resultados de oportunidad por especialidad}

En la Figura 2 se muestran los resultados de oportunidad por especialidad. En esta figura, se observa que el valor promedio del indicador de oportunidad difiere entre especialidades. 


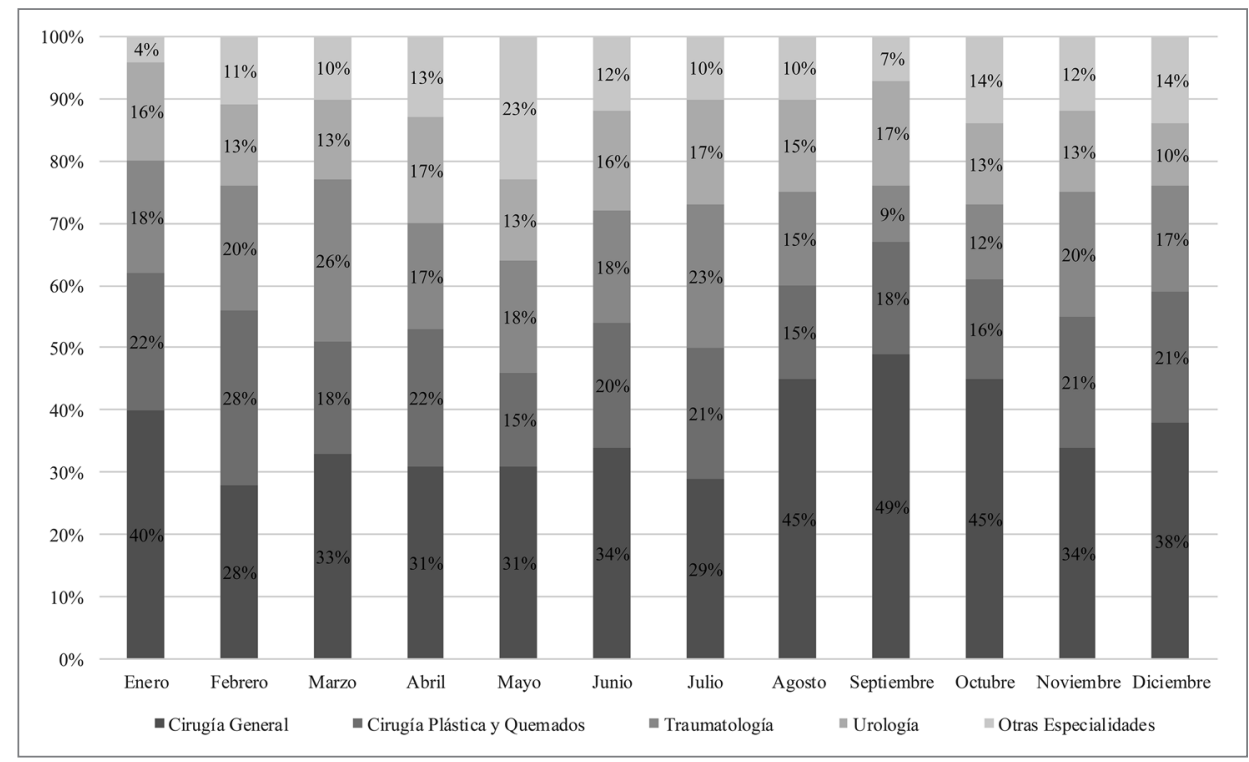

Figura 1. Asignación de pabellones por especialidad

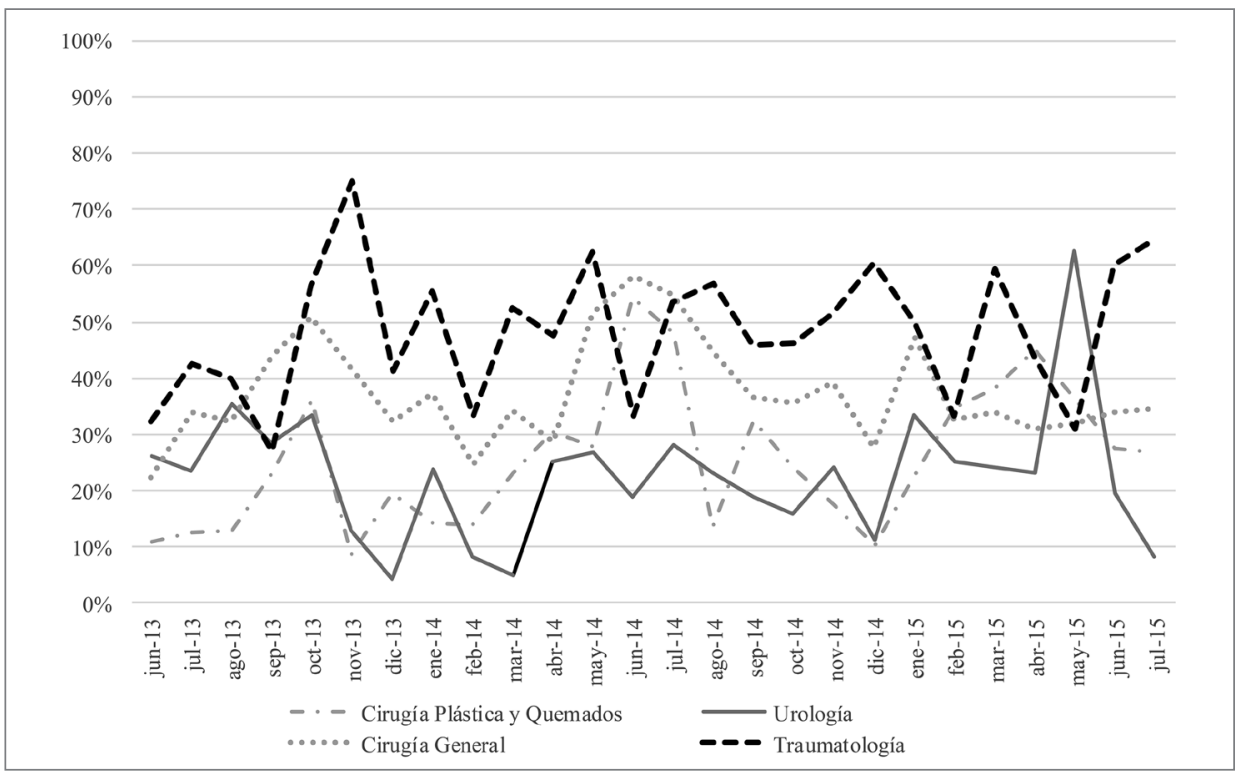

Figura 2. Oportunidad de atención por especialidad.

El indicador de oportunidad de Urología es el menor de las especialidades y su comportamiento es relativamente constante en el tiempo; en el caso de Traumatología, este indicador tiene el mayor valor promedio y generalmente fluctúa entre 30$60 \%$. La diferencia entre sus valores promedio significa que ambas especialidades tienen políticas distintas pero consistentes de selección pacientes, lo cual puede explicarse por distintos criterios de gestión clínica de las listas de espera.

\section{Resultados de justicia por especialidad}

La Figura 3 muestra los resultados de justicia por especialidad. En esta figura se puede notar que el valor promedio del indicador de justicia difiere entre especialidades, aunque su comportamiento es estable en el tiempo. Es interesante observar el incremento del indicador de justicia en Cirugía General y Traumatología en los últimos 12 meses.

En contraste con la situación observada en el indicador de oportunidad, la justicia en Urología 


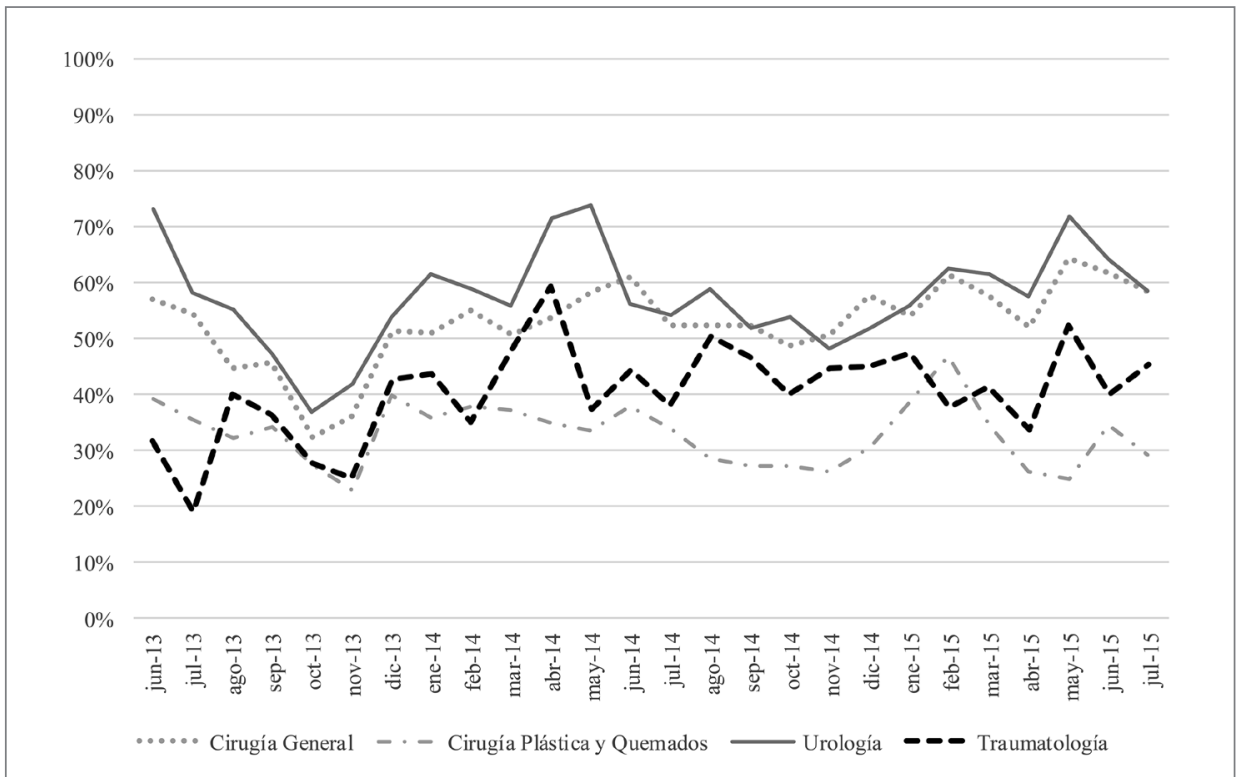

Figura 3. Justicia en la atención de especialidades.

es, en promedio, $17 \%$ mayor que en Traumatología. Este resultado muestra que la política de selección de pacientes en Urología es más congruente con el concepto de justicia igualitaria que la política de Traumatología.

\section{Discusión}

\section{Priorización de listas de espera}

El diseño e implementación de modelos de categorización de pacientes y priorización de listas de espera, ha permitido transparentar y facilitar la selección de pacientes para cirugía electiva.

El método de categorización contiene criterios explícitos que dificultan su manipulación. Otras ventajas de esta propuesta son: la incorporación de mejores prácticas a la decisión de categorización, consistencia en la categorización de pacientes entre especialistas, y compatibilidad con otras estrategias ministeriales. Una desventaja de los métodos presentados es que la definición de criterios explícitos de categorización y priorización requiere de un extenso trabajo con todos los equipos de cirujanos del hospital.

La perspectiva igualitaria utilizada para categorizar pacientes en este hospital corresponde a una decisión de los equipos médicos, enfocada en el riesgo del paciente. Sin embargo, el método propuesto permite incorporar la perspectiva utilitaria si se considera relevante y existe la información disponible para hacerlo.

Desde el punto de vista del modelo de atención, el hecho que el cirujano sea quien realiza el ingreso de pacientes a la lista de espera permite que la generación y gestión de las listas sea un proceso clínico y no administrativo, que comienza con la indicación quirúrgica en el box médico y finaliza con el protocolo en el pabellón.

\section{Modelo de gestión de listas de espera}

Esta investigación se sitúa en un contexto de la salud pública en Chile donde las políticas públicas han logrado subsanar la cobertura de la atención en el sistema y se encuentran en proceso de garantizar oportunidad.

El presente trabajo muestra que la oportunidad permite evaluar la atención de un paciente. Sin embargo, no proporciona información sobre el contexto en el cual esta persona es atendida, es decir, no evalúa esta decisión en relación al resto de los pacientes en espera. Esto último es fundamental en sistemas de salud públicos que buscan equidad en la atención.

Para avanzar en este sentido, se propone un modelo de gestión de listas de espera que incluya la justicia de forma complementaria a la oportunidad de atención. Un modelo definido de esta forma permite evaluar la gestión de listas 
de espera desde una perspectiva social, en base a equidad de acceso y resultado sanitario, pero sin perder de vista la mirada paciente-céntrica. Los resultados de esta experiencia muestran que los hospitales deberían mantener un balance entre estos indicadores y enfocarse en el desarrollo de iniciativas que mejoren la oportunidad y justicia de forma simultánea.

Dentro del hospital, el uso de este modelo puede expandirse a otros procesos con lista de espera, como la atención ambulatoria u otros procedimientos no quirúrgicos.

El modelo propuesto contiene definiciones que pueden utilizarse para analizar la efectividad de políticas públicas de salud. Por ejemplo, la estrategia de lista de espera presidencial no necesariamente mejora el indicador de justicia, en tanto se operan pacientes en función de una fecha de ingreso y no en base a criterios clínicos. Por otro lado, si bien las garantías AUGE han permitido avanzar en oportunidad de atención, éstas pueden tener un efecto negativo en el indicador de justicia al incluir sólo un grupo de patologías en el programa. Sin prejuicio de lo anterior, esta propuesta es compatible con las GES, ya que permite la incorporación de los criterios de priorización a los tiempos máximos de espera definidos en el AUGE.

\section{Trabajos futuros}

Existe acuerdo entre los cirujanos del hospital en cuanto a la validez de los métodos presentados en este trabajo. No obstante, es necesario realizar una validación cruzada con otros métodos disponibles para concluir respecto de su efectividad.

La utilización de este modelo de gestión de listas de espera sienta las bases para el análisis de capacidad quirúrgica del hospital y de la asignación de pabellones a especialidades. Esto puede realizarse en función de la caracterización de los pacientes por especialidad. La ventaja de este enfoque es que sitúa la oportunidad de atención como eje central del diseño de capacidad.

Agradecimientos: Los autores agradecen el aporte de todos los funcionarios del HEGC, en particular a: Dra. Nelly Letelier, jefe de Urología; Dr. Pedro José López, cirujano Urólogo; Dra. Marcela Santos, jefe de Cirugía General; Dra. Jacqueline Yáñez, jefe de Cirugía Plástica y Quemados; Dr. Mauricio Vergara, jefe de Traumatología; Dr. Patricio Herrera, subjefe de Cirugía; Dr. Ricardo
Zubieta, jefe de Cirugía; y de la Srta. Doris Soto. Fue fundamental también el apoyo del Magíster en Ingeniería de Negocios con TI y del Centro de Inteligencia de Negocios de la Universidad de Chile.

\section{Referencias}

1. Siciliani L, Hurst J. Tackling excessive waiting times for elective surgery: a comparative analysis of policies in 12 OECD countries. Health policy 2005; 72 (2): 201-15.

2. Valente R, Testi A, Tanfani E, Fato M, Porro I, Santo M, et. al. A model to prioritize access to elective surgery on the basis of clinical urgency and waiting time. Bio Med Central health services research 2009; 9 (1): 1.

3. Curtis AJ, Russell CO, Stoelwinder JU, McNeil JJ. Waiting lists and elective surgery: ordering the queue. Medical Journal of Australia 2010; 192 (4): 217-20.

4. Mullen P. Prioritising waiting lists: how and why? European Journal of Operational Research 2003; 150 (1): 32-45.

5. De Belvis A, Marino M, Avolio M, Pelone F, Basso D, Dei Tos G, et al. Wait watchers: the application of a waiting list active management program in ambulatory care. International journal for quality in health care 2013; 25 (2): 205-12.

6. Hilkhuysen G, Oudhoff J, Rietberg M, Van der Wal G, Timmermans D. Waiting for elective surgery: a qualitative analysis and conceptual framework of the consequences of delay. Public Health 2005; 119 (4): 290-3.

7. Evans D, Price N. The Ethical Dimensions of the National Waiting Time Project. A Report prepared for the Health Funding Authority. Dunedin: Bioethics Centre, Dunedin School of Medicine, University of Otago, 1999.

8. Oudhoff J, Timmermans D, Rietberg M, Knol D, Van der Wal G. The acceptability of waiting times for elective general surgery and the appropriateness of prioritising patients. Bio Med Central health services research 2007; 7 (1): 32 .

9. Hadorn D, Holmes A. The New Zealand priority criteria project. Part 2: Coronary artery bypass graft surgery. BMJ: British Medical Journal 2007; 314 (7074): 135.

10. Testi A, Tanfani E, Valente R, Ansaldo L, Torre C. Prioritizing surgical waiting lists. University of Genova, Villa Scassi Hospital, Genova, Italy. Journal of Evaluation in Clinical Practice 2006; 14: 59-64.

11. Mahon J, Bourne R, Rorabeck C, Feeny D, Webster-Bogaert S. Health-related quality of life and mobility of patients awaiting elective total hip arthroplasty: a prospective study. Canadian Medical Association Journal 2002; 167: 1115-21. 
12. MacCormick A, Collecutt W, Parry B. Prioritizing patients for elective surgery: a systematic review. Australian and New Zealand Journal of Surgery 2003; 73 (8): 633-42.

13. Noseworthy T, McGurran J, Hadorn D \& the Steering, Committee of the Western Canada Waiting List Project. Waiting for scheduled services in Canada: development of priority-setting scoring system. Journal of Evaluation in Clinical Practice 2003; 9 (1): 23-31.

14. Davis B, Johnson S. Waiting List Prioritisation, Carmarthen and District NHS Trust, Mimeo 1999.

15. Russell C, Roberts M, Williamson T, McKercher J, Jolly S, McNeil J. Clinical categorization for elective surgery in Victoria. Australian and New Zealand Journal of Surgery 2003; 73 (10): 839-42.

16. Derrett S, Paul C, Herbison P, Williams H. Evaluation of explicit prioritisation for elective surgery: a prospective study. Journal of health services research \& policy 2002; 7 (1): 14-22.

17. Escobar A, Quintana J, González M, Bilbao A, Ibáñez B. Waiting list management: priority criteria or first-in first-out? A case for total joint replacement. Journal of evaluation in clinical practice 2009; 15 (4): 595-601.

18. Min D, Yih Y. An elective surgery scheduling problem considering patient priority. Computers \& Operations Research 2010; 37 (6): 1091-9.

19. Wolff P, Yarza MV, Quezada A, Contesse J, Apablaza S. Priorización basada en la oportunidad de los pacientes quirúrgicos electivos. En: II Congreso Latinoamericano de Salud Global. http://www.educa.saludpublica.uchile. cl/saludglobal_2013/es/Resumenes_Trabajos_Libres/ Experiencias_Innovadoras/investigacion_e_innovacion_en_salud_global/Priorizacion_basada_en_la_oportunidad.pdf (accedido el 21 de marzo de 2016). 